CERN-PH-TH 2007/144

\title{
On the Consistency of Coset Space Dimensional Reduction
}

\author{
A. Chatzistavrakidis ${ }^{1,2}$, P. Manousselis ${ }^{2,3}$, N. Prezas ${ }^{4}$ and G. Zoupanos ${ }^{2}$ \\ 1 Institute of Nuclear Physics, \\ NCSR DEMOKRITOS, \\ GR-15310 Athens, Greece \\ ${ }^{2}$ Physics Department, National Technical University of Athens, \\ GR-15780 Zografou Campus, Athens, Greece \\ ${ }^{3}$ Department of Engineering Sciences, University of Patras, \\ GR-26110 Patras, Greece \\ ${ }^{4} \mathrm{CERN} \mathrm{PH}-\mathrm{TH}$, \\ 1211 Geneva, Switzerland \\ Email: cthan@mail.ntua.gr, pman@central.ntua.gr, george.zoupanos@cern.ch, \\ nikolaos.prezas@cern.ch
}

\begin{abstract}
In this letter we consider higher-dimensional Yang-Mills theories and examine their consistent coset space dimensional reduction. Utilizing a suitable ansatz and imposing a simple set of constraints we determine the four-dimensional gauge theory obtained from the reduction of both the higher-dimensional Lagrangian and the corresponding equations of motion. The two reductions yield equivalent results and hence they constitute an example of a consistent truncation.
\end{abstract}




\section{Introduction}

The first attempt to unify interactions through higher dimensions and dimensional reduction was made by Nordström [1, 2] even earlier than the celebrated work of Kaluza and Klein [3, 4]. The Kaluza-Klein proposal was to unify gravity and electromagnetism by considering five-dimensional gravity dimensionally reduced on a circle down to four dimensions. Compactification of higher-dimensional gravity was studied further by Pauli [5], who assumed a 2-sphere as the extra-dimensional space, and later by DeWitt [6]. One implication of considering internal spaces with non-abelian isometry is that Yang-Mills fields appear naturally in lower dimensions 1 . As a result, a revival of interest in the Kaluza-Klein proposal was inspired by the hope that a geometrical unification of gravity with the other observed non-abelian gauge interactions could be achieved [8].

However, this ambitious program met also serious obstacles. The most serious, plaguing all attempts of constructing realistic theories, was the inability to obtain chiral fermions in four dimensions [9]. Fortunately, there is a very interesting resolution to this problem achieved by adding Yang-Mills fields to the original higher-dimensional theory [10, 11]. Introducing non-Abelian gauge fields in higher dimensions is also welcome for another reason; it provides a potential unification of the low-energy gauge interactions as well as of the gauge and the Higgs fields. Concerning the latter we should recall that the celebrated Standard Model of elementary particle physics has obvious limitations due to the presence of a plethora of free parameters mostly related to the ad-hoc introduction of the Higgs and Yukawa sectors in the theory.

The Coset Space Dimensional Reduction (CSDR) [12, 13, 14] was suggesting from the beginning that a unification of the gauge and Higgs sectors can be achieved using higher dimensions. In the CSDR scheme one assumes that the space-time is of the form $M^{D}=$ $M^{4} \times S / R$ with $S / R$ being a homogeneous coset space. Then a gauge theory with gauge group $G$ defined on $M^{D}$ can be dimensionally reduced to $M^{4}$ in an elegant way by employing the symmetries of $S / R$. The resulting four-dimensional gauge group is a subgroup of $G$ and the four-dimensional gauge and Higgs fields are simply the surviving components of the gauge fields of the pure higher-dimensional gauge theory.

Upon introducing fermions [10] the four-dimensional Yukawa and fermion-gauge interactions find a unified description in the gauge interactions of the higher-dimensional theory. In this vein, another appealing feature of the CSDR is the observation that utilizing nonsymmetric coset spaces leads to softly broken supersymmetric theories [15]. Let us finally mention that a first step towards embedding the CSDR in string theory was taken in ref. [16] where it was shown that six-dimensional nearly-Kähler coset spaces provide internal spaces

\footnotetext{
${ }^{1}$ A nice review of these developments can be found in ref. [7].
} 
for supersymmetric heterotic string compactifications in the presence of fluxes and condensates.

An important point, which has been raised by a number of authors (e.g. [17]), concerns the consistency of the various reduction schemes. It is therefore natural to ask if the CSDR is consistent. A consistent reduction scheme of higher-dimensional theories over a unimodular group manifold $S$ was discovered by Scherk and Schwarz using the ansatz that the metric and the various spacetime fields are invariant under either the left or the right translations of the group [18]. A similar ansatz was employed in ref. [19] for the reduction on a coset space $S / R$ and one of the goals of this work was to obtain the CSDR constraints from the EinsteinYang-Mills equations of motion. It should be emphasized that there is no known recipe for obtaining consistent coset reductions neither a systematic understanding of the conditions under which such a reduction occurs. In the very few existing examples of consistent coset reductions there is very little conceptual understanding of the underlying reasons for the consistency but the detailed calculation itself. Some examples can be found in refs. [17, 21] and in the references therein.

Recently the concepts of truncation and reduction as well as the criteria for consistency were re-examined and analyzed [20, 7]. The upshot is that if we consider a theory described by a Lagrangian $\mathcal{L}$ as the starting point, then, for a certain number of dimensions of spacetime we can perform a truncation of $\mathcal{L}$ by essentially two methods:

(a) first type: ordinary Kaluza-Klein dimensional reduction where only the massless modes are kept,

(b) second type: by introducing constraints that reduce the number of independent fields - or field components - defining the theory.

These two procedures are usually applied simultaneously and constitute the concept of dimensional reduction. In both cases we are performing a truncation in the field content of the theory, either because Kaluza-Klein modes are eliminated in the dimensional reduction or because some field components are rendered redundant due to the presence of constraints. The consistency of such a truncation is decided by examining whether solutions of the equations of motion of the reduced Lagrangian $\mathcal{L}_{R}$ are still solutions of the equations of motion of the original Lagrangian $\mathcal{L}$. This property is expressed graphically as the commutativity of the following diagram:

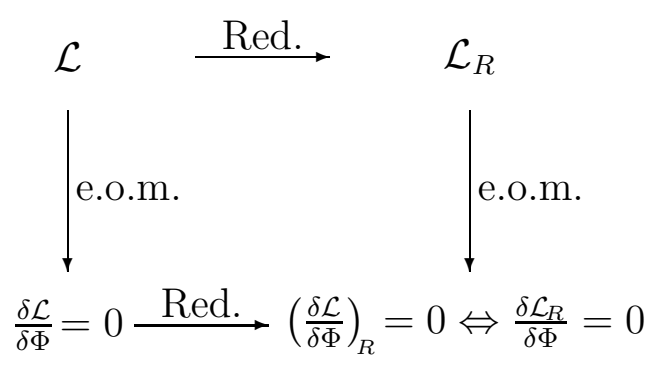


A proper definition is the following: a truncation is said to be consistent when its implementation at the level of the variational principle agrees with its implementation at the level of the equations of motion, i.e., if these operations commute: first truncate the Lagrangian and then obtain the equations of motion (e.o.m.) or first obtain the e.o.m. and then truncate them.

Concerning truncations of the second type, i.e. driven by the introduction of constraints, the outcome of the analysis of [20, 7] is that a mechanism parallel to that of the DiracBergmann theory of constrained systems applies. In general, the presence of secondary constraints - dynamically derived from the original (primary) ones - is a typical obstruction to the consistency of the truncation. In this letter we prove that the coset space dimensional reduction of [13] is an example of a truncation of the second type and that it is consistent in the sense explained earlier. In particular no new constrains are needed for the reduction of the higher-dimensional equations of motion besides the ones necessary for the proper reduction of the Lagrangian.

\section{Reduction on coset spaces: brief reminder}

\subsection{Geometry of coset spaces}

The geometry of coset spaces $S / R$ relevant for our purposes is presented in refs. [22, 23]. Let the coordinates of the Lie group $S$ be $\left(y^{a}, z^{i}\right)$ with $y^{a}$ being the coset coordinates and $z^{i}$ being the coordinates of the $R$ subgroup. Then a group element $s \in S$ can be represented as $s \sim e^{y^{a} Q_{a}} e^{z^{i} Q_{i}}$ and a coset representative is $L(y)=e^{y^{a} Q_{a}}$. The Maurer-Cartan 1-form is defined by $e(y)=L^{-1}(y) d L$ and is the analogue of the left-invariant 1-form on a Lie group $S$. It takes values in $\operatorname{Lie}(S)$, i.e. the Lie algebra of $S$ :

$$
e(y)=e^{A} Q_{A}=e^{a} Q_{a}+e^{i} Q_{i}
$$

where $A$ is a group index, $e^{a}$ is the coframe and $e^{i}$ is the $R$-connection. The latter can be expanded in coset vielbeins as $e^{i}=e_{a}^{i}(y) e^{a}$. The exterior derivative of the Maurer-Cartan 1 -form is

$$
d e=d\left(L^{-1} d L\right)=-e \wedge e=-[e, e]
$$

from which we can easily prove that

$$
d e^{A}=-\frac{1}{2} f_{B C}^{A} e^{B} \wedge e^{C}
$$

We will assume, for reasons analyzed in detail in ref. [22], that the coset is reductive. That means that the commutation relations obeyed by the generators of $S$ are not the most 
general ones but they take the form

$$
\begin{aligned}
{\left[Q_{i}, Q_{j}\right] } & =f_{i j}^{k} Q_{k} \\
{\left[Q_{i}, Q_{a}\right] } & =f_{i a}^{b} Q_{b} \\
{\left[Q_{a}, Q_{b}\right] } & =f_{a b}^{c} Q_{c}+f_{a b}^{i} Q_{i}
\end{aligned}
$$

implying that $f_{b i}^{j}=0$. Now (2.3) can be written as

$$
\begin{gathered}
d e^{a}=-\frac{1}{2} f_{b c}^{a} e^{b} \wedge e^{c}-f_{b i}^{a} e^{b} \wedge e^{i}, \\
d e^{i}=-\frac{1}{2} f_{a b}^{i} e^{a} \wedge e^{b}-\frac{1}{2} f_{j k}^{i} e^{j} \wedge e^{k}
\end{gathered}
$$

and from eq. (2.5) we can obtain the Maurer-Cartan equations for the coset vielbeins

$$
d e^{a}=-\frac{1}{2} C_{b c}^{a}(y) e^{b} \wedge e^{c}, \quad C_{b c}^{a}=f_{b c}^{a}-2 e_{[b}^{i} f_{c] i}^{a} .
$$

It is straightforward to verify the following identities that we will frequently use in the ensuing

$$
\begin{aligned}
& e^{a} \wedge *_{d} e^{b}=\delta^{a b} \text { vol }_{d}, \\
& d *_{d} e^{a}=C_{b a}^{b}(y) v_{d}, \\
& e^{a} \wedge *_{d}\left(e^{b} \wedge e^{c}\right)=\delta^{a c} *_{d} e^{b}-\delta^{a b} *_{d} e^{c} \\
& d *_{d}\left(e^{a} \wedge e^{b}\right)=\left(* e^{a}\right) C_{c b}^{c}-\left(* e^{b}\right) C_{c a}^{c}-C_{a b}^{c}\left(* e^{c}\right) .
\end{aligned}
$$

In these formulas $v o l_{d}$ is the volume form of the coset. Notice that the tangent coset indices $a, b, \ldots$ are raised and lowered by $\delta_{a b}$ and hence upper and lower such indices are equivalent.

Finally, let us mention that there exist $S$-invariant metrics on $S / R$. An example is

$$
g(y)=\gamma_{a b} e^{a}(y) e^{b}(y)
$$

where $\gamma_{a b}$ is the Killing metric of the group restricted on the coset $S / R$. The Killing vectors associated with the left isometry group $S$ will be denoted by $X_{I}$.

\subsection{Reduction on group manifolds and coset spaces}

Before we describe the Coset Space Dimensional Reduction (CSDR) scheme, let us recall that the ansatz for the Scherk-Schwartz reduction [18] of higher-dimensional gauge field $A$ on a group manifold $S$ is

$$
A=A_{\mu} d x^{\mu}+A_{I}(x) e^{I}(y)
$$


with $I=1, \ldots, \operatorname{dim} S$ and $e^{I}$ being the left-invariant 1-forms. Hence, in this type of reduction on group manifolds we keep only the $G_{L}$ singlets under the full isometry group $G_{L} \times G_{R}$ of $G$. This truncation can be described by the following invariance condition:

$$
\mathcal{L}_{X^{I}} A=0
$$

with $X^{I}$ being the Killing vectors dual to the right-invariant 1-forms2 The Scherk-Schwarz reduction of the metric is performed by enforcing a similar invariance condition

$$
\mathcal{L}_{X^{I}} g_{M N}=0 .
$$

In the CSDR one allows a generalized invariance condition

$$
\mathcal{L}_{X^{I}} A=D W_{I}
$$

with $W_{I}$ being a gauge transformation parameter associated to the Killing vector $X_{I}$. The invariance condition for the reduction of the metric is the same as in the group manifold case since the metric is gauge singlet. The generalized invariance condition

$$
\mathcal{L}_{X^{I}} A=i_{X^{I}} d A+d i_{X^{I}} A=D W_{I}=d W_{I}+[A, W]
$$

together with the consistency condition

$$
\left[\mathcal{L}_{X^{I}}, \mathcal{L}_{X^{J}}\right]=\mathcal{L}_{\left[X^{I}, X^{J}\right]}
$$

impose constraints on the gauge field. The detailed analysis of the constraints (2.17) and (2.18) given in refs. [13, 14] provides us with the four-dimensional unconstrained fields as well as with the residual gauge invariance of the lower-dimensional theory.

\section{CSDR of Yang-Mills theory}

\subsection{Gravity background}

The question of compatibility of the CSDR constraints with the equations of motion was originally raised in [19]. In this letter we revisit this question in light of the recent analysis of [20, 7].

We begin by examining a $D$-dimensional Einstein-Yang-Mills Lagrangian

$$
\mathcal{L}=\hat{R} *_{D} \mathbf{1}-\frac{1}{2} \operatorname{Tr} \hat{F}_{(2)} \wedge *_{D} \hat{F}_{(2)}-\lambda_{(D)} *_{D} \mathbf{1}
$$

\footnotetext{
${ }^{2}$ Recall that the right-invariant vector fields generate left-translations.
} 
where

$$
\hat{F}_{(2)}=d \hat{A}_{(1)}+\hat{A}_{(1)} \wedge \hat{A}_{(1)}
$$

is a non-Abelian gauge field strength taking values in the Lie algebra of a group $G$. $\hat{R}$ is the curvature scalar and $\lambda_{(D)}$ is the cosmological constant in $D$-dimensions. We distinguish the higher-dimensional fields from the four-dimensional ones by putting hats on them. The equations of motion are

$$
\begin{aligned}
& \hat{R}_{M N}=\frac{1}{2} \operatorname{Tr}\left(\hat{F}_{M N}^{2}-\frac{1}{8} \hat{F}^{2} \hat{g}_{M N}\right)+\frac{1}{4} \lambda_{(D)} \hat{g}_{M N}, \\
& \hat{D}\left(*_{D} \hat{F}_{(2)}\right)=0 .
\end{aligned}
$$

We will perform a reduction on a spacetime of the form $M_{4} \times S / R$ where $M_{4}$ is the fourdimensional Minkowski spacetime and $S / R$ is a compact coset space of dimension $d=D-4$. This background is described by a metric of the form 3

$$
g_{(D)}=\eta_{m n} e^{m} e^{n}+\gamma_{a b} e^{a} e^{b}
$$

where $\eta_{m n}$ is the flat Minkowski metric and $\gamma_{a b}$ is an $S$-invariant metric on the coset $S / R$ satisfying (2.15). From the metric (3.4) and using the vielbeins constructed from the MaurerCartan 1-form on the coset $S / R$ we can compute the Ricci curvature and Ricci scalar. The Einstein equations (3.2) take the form

$$
\frac{1}{4} \operatorname{Tr} F_{a b} F^{a b}=\lambda_{(D)}
$$

and

$$
\operatorname{Tr} F_{a b}^{2}=R_{(d)} \gamma_{a b}
$$

where $\lambda_{D}$ is the higher-dimensional cosmological constant and $R_{(d)}$ is the scalar curvature of the internal space. Let us note that the complete elimination of the Kaluza-Klein gauge fields as well as of the scalar moduli is a well-known consistent truncation of gravity on coset spaces [20]. We plan to examine a more general reduction scheme of gravity on cosets in future work [24].

\subsection{Reduction of the action}

The reduction ansatz for the gauge fields is

$$
\hat{A}^{\tilde{I}}(x, y)=A^{\tilde{I}}(x)+\chi_{\alpha}^{\tilde{I}}(x, y) d y^{\alpha},
$$

\footnotetext{
${ }^{3}$ We denote tangent spacetime indices by $m, n, \ldots$ and curved ones by $\mu, \nu, \ldots$, tangent coset indices by $a, b, \ldots$ and curved ones by $\alpha, \beta, \ldots$, while for the full group $S$ tangent indices are denoted by $I, J, \ldots$ and curved ones by $A, B, \ldots$. Indices on the subgroup $\mathrm{R}$ are denoted by $i, j, \ldots$ Finally, the spacetime coordinates are $x^{\mu}$ while those of the coset space are $y^{\alpha}$.
} 
where

$$
\chi_{\alpha}^{\tilde{I}}(x, y)=\phi_{A}^{\tilde{I}}(x) e_{\alpha}^{A}(y)
$$

and $\tilde{I}$ is a Lie algebra index $\tilde{I}=1, \ldots, \operatorname{dim} G$. This formally looks exactly the same as the reduction ansatz on group manifolds. The objects $\phi_{A}^{\tilde{I}}(x)$ take values in the Lie algebra of $G$ and are coordinate scalars in four dimensions that can be interpreted as Higgs fields. The ansatz solves the generalized symmetry condition (2.16) provided we can embed $R$ in $G$ as explained in refs. [13, 14].

Next we substitute the above ansatz into the action and subsequently into the equations of motion. The gauge field can be written as

$$
\hat{A}^{\tilde{I}}=A^{\tilde{I}}+\phi_{A}^{\tilde{I}} e^{A}
$$

and the corresponding field strength is

$$
\hat{F}^{\tilde{I}}=d \hat{A}^{\tilde{I}}+\frac{1}{2} f_{\tilde{J} \tilde{K}}^{\tilde{I}} \hat{A}^{\tilde{J}} \wedge A^{\tilde{K}}
$$

with $f_{\tilde{J} \tilde{K}}^{\tilde{I}}$ being the structure constants of the higher-dimensional gauge group $G$.

Using (2.3) we compute

$$
\hat{F}^{\tilde{I}}=F^{\tilde{I}}+D \phi_{A}^{\tilde{I}} \wedge e^{A}-\frac{1}{2} F_{A B}^{\tilde{I}} e^{A} \wedge e^{B},
$$

where

$$
\begin{aligned}
F^{\tilde{I}} & =d A^{\tilde{I}}+\frac{1}{2} f_{\tilde{J} \tilde{K}}^{\tilde{I}} A^{\tilde{J}} \wedge A^{\tilde{K}}, \\
D \phi_{A}^{\tilde{I}} & =d \phi_{A}^{\tilde{I}}+f_{\tilde{J} \tilde{K}}^{\tilde{I}} A^{\tilde{J}} \phi_{A}^{\tilde{K}} \\
F_{A B}^{\tilde{I}} & =f_{A B}^{C} \phi_{C}^{\tilde{I}}-\left[\phi_{A}, \phi_{B}\right]^{\tilde{I}} .
\end{aligned}
$$

Note that we obtain (3.11), which admits gauge invariant constraints, provided we use the ansatz (3.9) . Had we used an ansatz of the form $\hat{A}^{\tilde{I}}(x, y)=A^{\tilde{I}}(x)+\phi_{a}^{\tilde{I}}(x) e^{a}$, then instead of structure constants $f_{B C}^{A}$ we would have obtained the tensors $C_{b c}^{a}(y)$ which are functions of $y$.

Next we will insert eq. (3.11) in (3.1) therefore obtaining the effective four-dimensional action and the CSDR constraints. To compute the higher-dimensional Yang-Mills action

$$
S=-\frac{1}{2} \int \operatorname{Tr} \hat{F} \wedge *_{D} \hat{F}
$$

we need to Hodge-dualize eq. (3.11) to

$$
*_{D} \hat{F}^{\tilde{I}}=*_{4} F^{\tilde{I}} \wedge \operatorname{vol}_{d}+*_{4} D \phi_{A}^{\tilde{I}} \wedge *_{d} e^{A}-\frac{1}{2} F_{A B} v o l_{4} \wedge *_{d}\left(e^{A} \wedge e^{B}\right) .
$$


In this formula $v l_{4}$ is the volume form of the four-dimensional spacetime while $*_{4}$ and $*_{d}$ denote the Hodge duality operation in spacetime and the coset respectively.

Inserting (3.11) and (3.16) in (3.15) we obtain the four-dimensional Lagrangian

$$
\mathcal{L}=-\frac{1}{2} \operatorname{Tr} F \wedge *_{4} F+\frac{1}{2} \operatorname{Tr} D \phi_{a} \wedge *_{4} D \phi_{a}-\frac{1}{4} F_{a b} F^{a b} v_{0 l}
$$

provided we impose the CSDR constraints:

$$
D \phi_{i}^{\tilde{I}}=F_{a i}^{\tilde{I}}=F_{i j}^{\tilde{I}}=0 .
$$

Explicitly the constraints (3.18) read 4

$$
\begin{aligned}
& F_{i b}=f_{i b}{ }^{c} \phi_{c}-\left[\phi_{i}, \phi_{b}\right]=0, \\
& F_{i j}=f_{i j}{ }^{k} \phi_{k}-\left[\phi_{i}, \phi_{j}\right]=0,
\end{aligned}
$$

as well as

$$
\left[A_{\mu}, \phi_{i}\right]=0
$$

The last equation comes from $D \phi_{i}^{\tilde{I}}$ assuming constant values for the scalars $\phi_{i}^{\tilde{I}}$ as dictated by Lorentz invariance. Notice that $F_{a b}$ depends on $\phi_{i}$ and in (3.17) the latter are set to their constant values which are determined by (3.20).

\subsection{Four-dimensional gauge group and spectrum}

At this point let us briefly explain how the above constraints determine the four-dimensional gauge group and the spectrum. Eq. (3.20) implies that the extra components $\phi_{i}$ are generators of $R$. Furthermore we assume that $R$ is a Lie subgroup of $G$. Then from (3.21) we conclude that the four-dimensional gauge fields $A_{\mu}$ have to commute with the generators of the $R$ subgroup of $G$. In other words, the gauge group in four dimensions $H$ is the centralizer of the embedding of $R$ in $G: H=C_{G}(R)$.

The fields $\phi_{a}(x)$ are scalars in four dimensions. From (3.19) we see that these fields transform under $R$ as a vector $v$

$$
S \supset R, \quad \operatorname{adj} S=\operatorname{adj} R+v,
$$

and furthermore that the $\phi_{a}(x)$ are intertwining operators connecting induced representations of $R$ acting on $G$ and $S / R$. This implies, exploiting Schur's lemma, that the transformation properties of the fields $\phi_{a}$ under $H$ can be deduced if we express the adjoint representation of $G$ in terms of $R \times H$ :

$$
G \supset R, \quad \operatorname{adj} G=(\operatorname{adj} R, 1)+(1, \operatorname{adj} H)+\sum\left(r_{i}, h_{i}\right) .
$$

\footnotetext{
${ }^{4}$ In order to avoid cluttering we will occasionally suppress the Lie algebra indices.
} 
Then if $v=\sum s_{i}$, where each $s_{i}$ is an irreducible representation of $R$, we obtain an $h_{i}$ multiplet of scalars $\phi_{a}$ for every pair $\left(r_{i}, s_{i}\right)$ with $r_{i}$ and $s_{i}$ being identical irreducible representations of $R$.

\subsection{Reduction of the equations of motion}

Next we examine the reduction of the higher-dimensional equations of motion. Since we have imposed constraints the consistency of this reduction is not guaranteed by the consistency of the reduction of the higher-dimensional Lagrangian [20]. Our final result will be that the diagram presented in the introduction is commutative, thereby proving the consistency of the CSDR scheme.

The higher-dimensional Yang-Mills equation is

$$
\hat{D} *_{D} \hat{F}^{\tilde{I}}=\hat{d} *_{D} \hat{F}^{\tilde{I}}+f_{\tilde{J} \tilde{K}}^{\tilde{I}} \hat{A}^{\tilde{J}} \wedge *_{D} \hat{F}^{\tilde{K}}=0 .
$$

Substituting the reduction ansatz (3.9, 3.16) into (3.24) we obtain

$$
D *_{4} F^{\tilde{I}} \wedge \operatorname{vol}_{d}-f_{\tilde{J} \tilde{K}}^{\tilde{I}} \phi_{A}^{\tilde{J}} *_{4} D \phi_{B}^{\tilde{K}} \wedge e^{A} \wedge *_{d} e^{B}-*_{4} D \phi_{A}^{\tilde{I}} \wedge d *_{d} e^{A}=0
$$

and

$$
D *_{4} D \phi_{A}^{\tilde{I}} \wedge *_{d} e^{A}-\frac{1}{2} f^{\tilde{I}} \tilde{J}_{\tilde{K}} \phi_{A}^{\tilde{J}} F_{B C}^{\tilde{K}} v o l_{4} \wedge e^{A} \wedge *_{d}\left(e^{B} \wedge e^{C}\right)-\frac{1}{2} F_{B C}^{\tilde{I}} v o l_{4} \wedge d *_{d}\left(e^{B} \wedge e^{C}\right)=0 .
$$

Using these relations and imposing the same constraints (3.18) we used earlier in the reduction of the Lagrangian, we obtain the equations of motion for the four-dimensional gauge fields and scalars:

$$
\begin{aligned}
D *_{4} F^{\tilde{I}} & =f_{\tilde{I} \tilde{K}}^{\tilde{I}} \phi_{a}^{\tilde{J}} *_{4} D \phi_{a}^{\tilde{K}}, \\
D *_{4} D \phi_{a}^{\tilde{I}} & =-\left(f_{\tilde{J} \tilde{K}}^{\tilde{I}} \phi_{c}^{\tilde{J}} F_{c a}^{\tilde{K}}+\frac{1}{2} F_{b c} f_{b c a}\right) v o l_{4} .
\end{aligned}
$$

These are the equations of motion for a Yang-Mills theory coupled to charged scalars with a non-trivial potential as described by the Lagrangian (3.17). Therefore the consistency of the truncation is verified.

In order to deduce (3.27) from (3.25) we have to show that the extra terms

$$
-f^{\tilde{I}} \tilde{\tilde{K}} \phi_{i}^{\tilde{J}} *_{4} D \phi_{b}^{\tilde{K}} \wedge e_{a}^{i} e^{a} \wedge *_{d} e^{b}-*_{4} D \phi_{a}^{\tilde{I}} \wedge d *_{d} e^{a}
$$

cancel out. First we notice that if $S$ is unimodular, i.e. it has traceless structure constants $f_{A B}^{A}=0$, and the coset is reductive we have $f_{a b}^{a}=0$. Then from (2.7) and (2.9) we find $d *_{d} e^{a}=-e_{b}^{i} f_{a i}^{b} v l_{d}$. Subsequently (3.29) becomes

$$
*_{4}\left(f_{a i}^{b} D \phi_{a}^{\tilde{I}}-f_{\tilde{I} \tilde{K}}^{\tilde{I}} \phi_{i}^{\tilde{J}} D \phi_{b}^{\tilde{K}}\right) e_{b}^{i} \wedge v l_{d}=*_{4}\left(D F_{i b}^{\tilde{I}}\right) e_{b}^{i} \wedge v l_{d}=0
$$


where we have used the constraints $D \phi_{i}^{\tilde{I}}=0$ and $F_{i b}=0$.

In order to derive (3.28) from (3.26) we write the terms

$$
-\frac{1}{2} f_{\tilde{J} \tilde{K}}^{\tilde{I}} \phi_{i}^{\tilde{J}} F_{b c}^{\tilde{K}} \operatorname{vol}_{4} \wedge e^{i} \wedge *_{d}\left(e^{b} \wedge e^{c}\right)-\frac{1}{2} F_{b c}^{\tilde{I}} v l_{4} \wedge d *_{d}\left(e^{b} \wedge e^{c}\right)
$$

and show that they equal the second term at the right-hand side of (3.28). Using (2.7), (2.10) and (2.11) we can re-write them as

$$
\begin{aligned}
& \operatorname{vol}_{4} \wedge * e^{c}\left[\left[\phi_{i}, F_{b c}\right]^{\tilde{I}} e^{i b}-F_{a b}^{\tilde{I}} e_{a}^{i} f_{b i}^{c}+F_{c b}^{\tilde{I}} e_{a}^{i} f_{b i}^{a}+\frac{1}{2} F_{a b}^{\tilde{I}} f_{a b}^{c}\right]= \\
& \operatorname{vol}_{4} \wedge * e^{c}\left[e_{b}^{i}\left(\left[\phi_{i}, F_{b c}\right]^{\tilde{I}}-\left(F_{b a}^{\tilde{I}} f_{a i}^{c}+F_{c a}^{\tilde{I}} f_{a i}^{b}\right)\right)+\frac{1}{2} F_{a b}^{\tilde{I}} f_{a b}^{c}\right] .
\end{aligned}
$$

The last term in this formula contributes to the equation of motion (3.28) while the rest cancel out. The cancellation is due to the identity

$$
\left[\phi_{i}, F_{a b}\right]^{\tilde{I}}=f_{i a}^{c} F_{c b}^{\tilde{I}}+f_{i b}^{c} F_{a c}^{\tilde{I}}
$$

that holds as a consequence of the constraint $\left[\phi_{i}, \phi_{a}\right]^{\tilde{I}}=f_{i b}{ }^{c} \phi_{c}^{\tilde{I}}$ and the definition of $F_{a b}^{\tilde{I}}=$ $f_{a b}^{c} \phi_{c}^{\tilde{I}}+f_{a b}^{i} \phi_{i}^{\tilde{I}}-\left[\phi_{a}, \phi_{b}\right]^{\tilde{I}}$, provided that we use the Jacobi identity and assuming that the structure constants $f_{A B C}$ of the Lie group $S$ are completely antisymmetric and that the coset $S / R$ is reductive.

\subsection{Bianchi identities}

To conclude our analysis we examine the higher-dimensional Bianchi Identity

$$
\hat{D} \hat{F}^{\tilde{I}}=\hat{d} \hat{F}^{\tilde{I}}+f_{\tilde{J} \tilde{K}}^{\tilde{I}} \hat{A}^{\tilde{J}} \wedge \hat{F}^{\tilde{K}}=0 .
$$

Substituting the reduction ansatz (3.9) and (3.11) into eq. (3.33) we obtain

$$
\begin{aligned}
& d F^{\tilde{I}}+f_{\tilde{J} \tilde{K}}^{\tilde{I}} A^{\tilde{J}} \wedge F^{\tilde{K}}=0, \\
& d D \phi_{A}^{\tilde{I}} \wedge e^{A}+f_{\tilde{J} \tilde{K}}^{\tilde{I}} A^{\tilde{J}} \wedge D \phi_{A}^{\tilde{K}} \wedge e^{A}-f_{\tilde{J} \tilde{K}}^{\tilde{I}} \phi_{A}^{\tilde{J}} F^{\tilde{K}} \wedge e^{A}=0, \\
& D F_{B C}^{\tilde{I}}=f_{B C}^{A} D \phi_{A}^{\tilde{I}}-2 f_{\tilde{J} \tilde{K}}^{\tilde{I}} \phi_{B}^{\tilde{J}} D \phi_{C}^{\tilde{K}}, \\
& \frac{1}{2}\left(F_{A B}^{\tilde{I}} f_{D C}^{A}-f_{\tilde{J} \tilde{K}}^{\tilde{I}} \phi_{B}^{\tilde{J}} F_{D C}^{\tilde{K}}\right) e^{D} \wedge e^{C} \wedge e^{B}=0 .
\end{aligned}
$$

These equations, after imposing the constraints (3.18) and utilizing the Jacobi identities for the structure constants, yield

- the Bianchi identity for the four-dimensional gauge field $D F=0$,

- the Bianchi identity for the four-dimensional scalars $D^{2} \phi=F \wedge \phi$. 


\section{Conclusions}

We have studied a particular dimensional reduction scheme of Yang-Mills theories with the understanding that there are no general recipes for consistent coset space reductions. Utilizing an ansatz inspired by an example of a consistent group manifold reduction, we have examined to what extent a coset reduction can be consistent. We demonstrated that upon imposing a simple set of constraints, which we identified as the constraints appearing naturally in the CSDR reduction scheme, one can reduce in a self-consistent manner both the higher-dimensional Lagrangian and the corresponding equations of motion. The next and substantially more involved step is to include gravity fluctuations. Work in this direction is currently in progress [24].

\section{Acknowledgements}

This work is supported by the EPEAEK program "Pythagoras" and co-funded by the European Union (75\%) and the Hellenic State (25\%). This work is also partially supported by the NTUA program for basic research "Karatheodoris". PM is supported by the Hellenic State Scholarship Foundation (I.K.Y.) and by the program Pythagoras I (89194).

\section{References}

[1] G. Nordstrom, "On a theory of electricity and gravitation," arXiv:physics/0702222].

[2] G. Nordstrom, "On the possibility of unifying the electromagnetic and the gravitational fields," Phys. Z. 15, 504 (1914) arXiv:physics/0702221].

[3] T. Kaluza, "On The Problem Of Unity In Physics," Sitzungsber. Preuss. Akad. Wiss. Berlin (Math. Phys. ) 1921, 966 (1921).

[4] O. Klein, "Quantum theory and five-dimensional theory of relativity," Z. Phys. 37, 895 (1926) [Surveys High Energ. Phys. 5, 241 (1986)].

[5] N. Straumann, "On Pauli's invention of non-Abelian Kaluza-Klein theory in 1953" , arXiv:gr-qc/ 0012054; L. O'Raifeartaigh and N. Straumann, "Early history of gauge theories and Kaluza-Klein theories, with a glance at recent developments", arXiv:hep-ph/9810524.

[6] B. S. De Witt, In C. and B. S. De Witt (ed.) "Relativity, Groups And Topology", Gordan and Breach, New York (1964). 
[7] J. M. Pons, "Dimensional reduction, truncations, constraints and the issue of consistency," arXiv:hep-th/0610268.

[8] R. Kerner, Ann. Inst. H. Poincaré 9 (1968) 143. Y. M. Cho, "Higher - Dimensional Unifications Of Gravitation And Gauge Theories," J. Math. Phys. 16, 2029 (1975). Y. M. Cho and P. G. O. Freund, "Nonabelian Gauge Fields In Nambu-Goldstone Fields," Phys. Rev. D 12, 1711 (1975). W. Mecklenburg, "The Kaluza-Klein Idea: Status And Prospects," Fortsch. Phys. 32, 207 (1984). D. Bailin and A. Love, "Kaluza-Klein theories," Rept. Prog. Phys. 50, 1087 (1987). A. Salam and J. A. Strathdee, "On KaluzaKlein Theory," Annals Phys. 141, 316 (1982).

[9] E. Witten, "Search for a realistic Kaluza-Klein theory," Nucl. Phys. B 186, 412 (1981). E. Witten, "Fermion Quantum Numbers In Kaluza-Klein Theory,"

[10] N. S. Manton, "Fermions And Parity Violation In Dimensional Reduction Schemes," Nucl. Phys. B 193, 502 (1981).

[11] G. Chapline and R. Slansky, "Dimensional Reduction And Flavor Chirality," Nucl. Phys. B 209, 461 (1982).

[12] E. Witten, "Some exact multipseudoparticle solutions of classical Yang-Mills theory," Phys. Rev. Lett. 38, 121 (1977).

[13] P. Forgacs and N. S. Manton, "Space-Time Symmetries In Gauge Theories," Commun. Math. Phys. 72, 15 (1980).

[14] D. Kapetanakis and G. Zoupanos, "Coset Space Dimensional Reduction Of Gauge Theories," Phys. Rept. 219, 1 (1992). Yu. A. Kubyshin, I. P. Volobuev, J. M. Mourao and G. Rudolph, "Dimensional reduction of gauge theories, spontaneous compactification and model building," Leipzig Univ. - KMU-NTZ-89-077 (89,REC.SEP.) 80p

[15] P. Manousselis and G. Zoupanos, "Dimensional reduction of ten-dimensional supersymmetric gauge theories in the $\mathrm{N}=1, \mathrm{D}=4$ superfield formalism," JHEP 0411, 025 (2004) arXiv:hep-ph/0406207|. P. Manousselis and G. Zoupanos, "Dimensional reduction over coset spaces and supersymmetry breaking," JHEP 0203, 002 (2002) arXiv:hep-ph/0111125. P. Manousselis and G. Zoupanos, "Soft supersymmetry breaking due to dimensional reduction over non-symmetric coset spaces," Phys. Lett. B 518, 171 (2001) arXiv:hep-ph/0106033. P. Manousselis and G. Zoupanos, "Supersymmetry breaking by dimensional reduction over coset spaces," Phys. Lett. B 504, 122 (2001) arXiv:hep-ph/0010141. 
[16] P. Manousselis, N. Prezas and G. Zoupanos, "Supersymmetric compactifications of heterotic strings with fluxes and condensates," Nucl. Phys. B 739, 85 (2006) arXiv:hep-th/0511122.

[17] M. Cvetic, G. W. Gibbons, H. Lu and C. N. Pope, "Consistent group and coset reductions of the bosonic string," Class. Quant. Grav. 20, 5161 (2003) arXiv:hep-th/0306043.

[18] J. Scherk and J. H. Schwarz, "How To Get Masses From Extra Dimensions," Nucl. Phys. B 153, 61 (1979).

[19] M. Surridge, "On stable, compactifying classical solutions of Einstein-Yang-Mills systems in (4+d) space-time dimensions," Z. Phys. C 37 (1987) 77.

[20] J. M. Pons and P. Talavera, "Consistent and inconsistent truncations. General results and the issue of the correct uplifting of solutions," Nucl. Phys. B 678, 427 (2004) arXiv:hep-th/0309079, J. M. Pons and P. Talavera, "Truncations driven by constraints: Consistency and conditions for correct upliftings," Nucl. Phys. B 703, 537 (2004) arXiv:hep-th/0401162.

[21] G. W. Gibbons and C. N. Pope, "Consistent S**2 Pauli reduction of six-dimensional chiral gauged Einstein-Maxwell supergravity," Nucl. Phys. B 697, 225 (2004) arXiv:hep-th/0307052.

[22] L. Castellani, L. J. Romans and N. P. Warner, "Symmetries Of Coset Spaces And Kaluza-Klein Supergravity," Annals Phys. 157, 394 (1984).

[23] L. Castellani, "On G/H geometry and its use in M-theory compactifications," Annals Phys. 287, 1 (2001) arXiv:hep-th/9912277.

[24] Work in progress, A. Chatzistavrakidis, P. Manousselis, N. Prezas and G. Zoupanos. 